ORIGINAL ARTICLE

\title{
Role of Diffusion Weighted Imaging \& Dynamic Contrast Enhanced Magnetic Resonance Imaging in Assessment of Asymmetric Breast Densities
}

\author{
Hazem M. El Shahat Mousa, Ghada El Sayied Abdulmonaem, and Dena Abd El Aziz El \\ Sammak
}

Corresponding author:

Hajer Abushaafa Elragig

Abdalla

Department of Radio-

diagnosis, Faculty of

Medicine -Zagazig

University, Egypt.

E-mail :

hajerabushaafa@gmail.com

Submit Date 2019-04-04

Revise Date 2019-06-11

Accept Date 2019-06-16

\section{ABSTRACT}

Objective: The aim of the study is to determine the diagnostic value of both DCE-MRI \& DW-MRI in assessment of cases with mammographic asymmetrical breast densities.

Methods: This study was carried out at Radiodiagnosis Department, Zagazig University Hospitals, on 29 patients with breast asymmetry. Diagnostic work up included Mammography, US, \& MRI.

Results: The study included 29 females with mammographic breast asymmetry, 17 were having global asymmetry, while 12 with focal asymmetry. $65.52 \%$ of asymmetry in this study was due to benign causes while $34.48 \%$ was due to malignant causes, DCE-MRI provided special characterization of lesions by their morphology \& dynamic enhancement with $90 \%$ sensitivity $84.21 \%$ specificity, \& $86.2 \%$ accuracy. DWI \& ADC value provided differentiation between malignant \& benign lesions, malignant lesions showed restricted diffusion with lower ADC values than benign lesions, the mean ADC value for benign lesions was $\left(1.4 \pm 0.45 \times 10^{-3} \mathrm{sec} / \mathrm{mm}^{2}\right)$, $\&$ the mean ADC value for malignant lesions was $\left(0.85 \pm 0.29 \times 10^{-3} \mathrm{sec} / \mathrm{mm}^{2}\right), \&$ the cutoff ADC value was $\left(<0.86 \times 10^{-3} \mathrm{sec} / \mathrm{mm}^{2}\right)$ for diagnosis of the malignant lesions. The DWI \& ADC value showed $90 \%$ sensitivity, $89.47 \%$ specificity, $\& 89.66 \%$ accuracy. By combining both protocols the overall MRI validity has been increased to be $90 \%$ sensitivity, $94.74 \%$ specificity, $90 \%$ PPV, $94.74 \%$ NPV, \& $93.1 \%$ accuracy.

Conclusion: Combining DWI to DCE-MRI in assessment of asymmetric breast densities provided better characterization \& differentiation of underlying lesions especially of probably benign, suspicious, \& highly suggestive malignant findings on conventional imaging, \& the combined MRI protocol increased the overall MRI accuracy.

Key words: Breast; DWI; DCE-MRI; Breast MRI

\section{INTRODUCTION}

A symmetrical breast densities detected in mammography may be due to an underlying mass, postoperative changes, hormonal replacement therapy, malpositioning or normal variations in breast tissue. ${ }^{[1,2]}$

The American College of Radiology (ACR), Breast Imaging Reporting and Data System (BI-RADS) defined four different types of asymmetric breast findings:

(1)Asymmetric Breast tissue: refers to a greater density of one breast than in the corresponding area in the contralateral breast.
(2)Densities seen in one projection: reflect a density seen in only one mammographic projection.

(3)Architectural distortion: refers to a focal area of breast tissue that appears distorted with no definable central mass. Spicules radiate from a common point, and there is an area of focal retraction and tethering of normal parenchyma.

(4)Focal asymmetric densities: refers to focal asymmetric densities that are seen on two mammographic views but cannot be accurately identified as a true mass. ${ }^{[3]}$ 
Although mammography \& ultrasonography are the most widely non-invasive imaging modalities used in screening \& evaluation of asymmetric breasts, these modalities may have limited sensitivity and specificity for the detection and diagnosis of breast lesions ${ }^{[4]}$

Magnetic resonance (MR) imaging has been increasingly used for accurate diagnosis of breast masses, particularly in cases in which mammography and breast sonography are inconclusive or yield discrepancies ${ }^{[5]}$ Dynamic contrast-enhanced breast MRI (DCE-MRI) has been reported to have high sensitivity in detecting breast cancer reportedly as high as $88-100 \%$, but its specificity may be in the range of $68-96 \% .^{[6]}$

Breast carcinomas generally show a faster and stronger signal intensity increase after a bolus injection of Gadolinium based contrast agent than most benign lesions. The limitation of specificity of DCE- MRI can be attributed to the fact that several benign breast lesions including fibroadenomas can also show strong contrast agent enhancement such that the signal intensity versus time curves considerably overlap those of breast carcinomas. ${ }^{\text {[7] }}$

Diffusion-weighted MRI produces in vivo images of biological tissues weighted with the local micro-structural characteristics of water diffusion. ${ }^{[8]}$ The Brownian motion of protons in bulk water produces the signal in DWI. The apparent diffusion coefficient (ADC) is a calculated value used to quantify Brownian motion. ${ }^{[9,}$ 10] The lower ADC values of malignant tissues are primarily attributed to higher cell density causing increased restriction of the extracellular matrix and increased fraction of signal coming from intracellular water. $^{[11,12]}$

\section{Patients \& Methods:}

This study was carried out at Radio diagnosis Department, Zagazig University Hospitals, during the period from April 2017 to October 2017. The present study was carried on 29 female patients who were referred to Radiodiagnosis department from General Surgery department \& who have asymmetric density findings on Mammography. Their ages ranged from 37 to 68 years with a mean of 49.7 years, all patients Subjected to dynamic MRI \&
DWI, histopathological diagnosis was performed in 20 cases \& cytology was done for 9 patients.

Written informed consent was obtained from all participants and the study was approved by the research ethical committee of Faculty of Medicine, Zagazig University. The work has been carried out in accordance with The Code of Ethics of the World Medical Association (Declaration of Helsinki) for studies involving humans.

\section{Patients:}

\section{A.Patient inclusion criteria:}

- Female patients with breast complaints.

- Mammography breast asymmetric density findings, which were classified on BI-RADS III, IV, or V.

- Premenopausal patients during the 2nd week of menstrual cycle, because during the proliferative phase the back ground enhancement of the normal breast tissue is low therefore abnormalities are better detected.

\section{B. Patient exclusion criteria:}

- Patients with BI-RADS I, II, \& VI.

- Patients unwilling to complete the study. Patients with severe renal impairment.

- Technical problems with the DWI sequences resulting from patient motion

- Patients with history of breast biopsy within 1 month, post-surgical breast scar, and patient with history of Hormonal replacement therapy (HRT).

\section{Methods:}

All patients were subjected to the following:

A. History: Including: Past medical History (breast complaints or surgeries, medications, Also any past history of a procedure that is considered as contraindication to MRI exam e.g., metallic aneurysm clips \& cardiac pacemakers). History of previous breast imaging. Family history of breast disease. Current clinical presentation (age, complaints, occupation \& residency). And investigations.

B. Clinical examination: It was focused on breast examination for any nipple retraction, skin thickening, erythema, palpable breast lumps or axillary lymph nodes, $\&$ any nipple discharge.

\section{Imaging:}

This includes the following: 
* Evaluation of the Mammography images in regard to the presence of asymmetric densities findings \& assessment of the complementary High Resolution Ultrasonography imaging, US was performed on Siemens machine by 7.5 $\mathrm{MHz}$ Superficial probe \& examination was done in radial \& antiradial planes.

* MRI Imaging: this was performed at Zagazig University Hospitals, using (Philips Achieva 1.5 $\mathrm{T}$ scanner, Netherlands), \& at a private center using (GE Healthcare 1.5 T), both with dedicated surface coil.

* Position: Patients lied in prone position with breasts hanging down into the coil \& centrally not touching them, with intravenous cannula in either right or left arm, the arms were placed beside the head $\&$ the head was supported with head holder \& the legs were supported on a pillow to help in toleration of the prone position.

* Contrast injection: $0.1 \mathrm{mmol} / \mathrm{kg}$ Gadolinium based contrast IV injection at rate of $2 \mathrm{ml} / \mathrm{sec}$, followed by 20-30 $\mathrm{ml}$ flush with power injector at same rate.

- Sequences: The examination started with 3 plane localizer to check positioning \& field of view. Parallel imaging.

- Axial T1 WI \& T2 WI \& Fat saturated T1 or T2, \& sagittal T2 fat saturated were obtained with slice thickness of $3 \mathrm{~mm} \& 1 \mathrm{~mm}$ interslice gap, \& standard FOV of $350 \mathrm{~mm}$ which had been adjusted according to patient's size.

- After injection of contrast agent several post contrast image acquisitions (Dynamic) were obtained at $(1.5,3,4.5 \& 6)$ minutes.

- DWI was performed prior contrast injection at b values $\left(0,300\right.$, and $\left.600 \mathrm{~s} / \mathrm{mm}^{2}\right) \&$ ADC map was drawn at $b$ value $\left(1000 \mathrm{~s} / \mathrm{mm}^{2}\right)$ with $3 \mathrm{~mm}$ slice thickness \& no gap. DWI examination lasted for 2 minutes.

- The overall examination lasted 15 mins.

\section{* MRI Image interpretation:}

- The images of Mammography and ultrasonography were revised prior MRI examination.

- MRI images were assessed \& evaluated for any lesions according to the ACR MR lexicon for the following:

- Site, size, number, Shape, margins of lesions \& Signal intensity of lesion on T1 WI, T2 WI, \& on Fat saturations sequence. Also signal intensities of lesion on DWI \& ADC map were compared for assessment of diffusion (facilitated or restricted).

- Post contrast enhancement pattern (mass, nonmass like, or both mass \& non mass like enhancement).

- Skin thickening, nipple retraction, pectoralis muscle invasion, \& Presence of enlarged axillary lymph nodes.

- ROI was placed within the lesion on the dynamic series, \& time intensity curve was automatically drawn by the software (persistent, plateau or wash out).

- The mean value within the ROI on ADC map was also measured by the scanner software which was multiplied by $10^{-3}$ to be equal to the ADC value.

\section{Data Analysis:}

Data were statistically analyzed \& presented in terms of frequencies \& percentage when appropriate, also terms of sensitivity, specificity, positive \& negative predicting values and accuracy were represented.

\section{RESULTS}

all patients in this study were subjected to dynamic MRI \& Diffusion weighted imaging, histopathological diagnosis was performed in 20 cases \& cytology was done for 9 patients as a standard of reference. In this study we found that the asymmetry type in $58.62 \%$ of patients was global asymmetry and in $41.38 \%$ was focal asymmetry (Table 1).

According to the pattern of enhancement the relation between lesion type $\&$ enhancement pattern was insignificant, but according to type of time intensity curve, the results were highly significant it demonstrated type I persistent and type II plateau are more in benign lesions but type III wash out was more in malignant tumors (Table 2).

Also the relation was significant in regard to the diffusion characteristics; as $90 \%$ of malignant lesions demonstrated restricted diffusion, while facilitated diffusion was more with benign lesions $89.5 \%$ (Table 3).

According to the ADC value there was a significant difference between ADC value measured in benign lesions and that of malignant tumors, the mean ADC value for 
benign lesions was $1.4 \times 10^{-3} \mathrm{sec} / \mathrm{mm}^{2}$ while the mean $\mathrm{ADC}$ value for malignant lesions was $0.85 \times 10^{-3} \mathrm{sec} / \mathrm{mm}^{2}$ and the Standard deviation for the benign $\&$ malignant lesions was $0.45 \&$ 0.29 respectively (Table 4 ).

to correlation between standard of reference \& DCE-MRI diagnosis the true positive cases were 16 benign and 9 malignant and only one malignant case diagnosed falsely by MRI as a benign while 3 benign cases diagnosed falsely as malignant While regarding the correlation between the diffusion weighted imaging \& gold standard the true positive cases were 17 cases benign and 9 cases malignant and only one malignant case diagnosed falsely by DWI \& ADC value as benign and two benign cases diagnosed falsely as malignant The gold standard confirmed that in this study the benign lesions were $65.52 \%$ compared to malignant lesions which were $34.48 \%$, the fibrocystic changes was the commonest benign lesion $31.58 \%$ followed by fibroadenomatoid changes in $21 \%$ of cases, while invasive ductal carcionoma (IDC) $40 \%$ was the most common malignant lesion followed by ductal carcinoma in situ \& inflammatory carcinoma $20 \%$ for both (supplementary file).

The study demonstrated insignificant relation between asymmetry type \& lesion type, but generally in this study $65.52 \%$ of the asymmetry is caused by benign lesions while $34.48 \%$ of the asymmetry is caused by malignant lesions (Table 1).

By using the combined MRI protocol with both DCE-MRI \& DWI with ADC value, the accuracy increased to reach $93.1 \%$, the sensitivity \& specificity were $90 \%$, PPV was $90 \&$ NPV was 94.74 (Table 5). The cutoff ADC value is $<0.86 \times 10-3 \mathrm{sec} / \mathrm{mm} 2$ with sensitivity of $87.5 \%$ \& specificity of $100 \%$ \& accuracy of $96.55 \%$ (Table 6) \& (Fig. 1).

Table (1): Benign \& malignant lesions in relation to asymmetry type.

\begin{tabular}{|c|c|c|c|c|c|c|c|c|}
\hline \multirow{2}{*}{$\begin{array}{c}\text { Type of } \\
\text { asymmetry }\end{array}$} & \multicolumn{2}{|c|}{$\begin{array}{c}\text { Benign } \\
(\mathrm{n}=19)\end{array}$} & \multicolumn{2}{c|}{$\begin{array}{c}\text { Malignant } \\
(\mathrm{n}=10)\end{array}$} & \multicolumn{2}{|c|}{ Total } & Fisher s exact & P value \\
\cline { 2 - 9 } & No & $\mathbf{\%}$ & $\mathbf{N o}$ & $\mathbf{\%}$ & $\mathbf{N o}$ & $\%$ & 1.13 \\
\hline Focal & 7 & 36.8 & 5 & 50 & 12 & 41.4 & \\
\hline Global & 12 & 63.2 & 5 & 50 & 17 & 58.6 & \\
\hline Total & 19 & 65.5 & 10 & 34.5 & 29 & 100 & \\
\hline
\end{tabular}

Table (2): Relation between lesion type \& time intensity curve type.

\begin{tabular}{|c|c|c|c|c|c|c|c|c|}
\hline \multirow[t]{2}{*}{ Curve type } & \multicolumn{2}{|c|}{$\operatorname{Benign}(n=19)$} & \multicolumn{2}{|c|}{ Malignant $(\mathrm{n}=10)$} & \multicolumn{2}{|c|}{ Total/curve type $(n=29)$} & \multirow{2}{*}{$\begin{array}{c}\text { Fisher s } \\
\text { exact }\end{array}$} & \multirow[t]{2}{*}{$P$ value } \\
\hline & No. & $\%$ & No. & $\%$ & No. & $\%$ & & \\
\hline Type I persistent & 9 & 47.37 & 0 & 0.0 & 9 & 31.03 & 14.21 & $<0.001 *$ \\
\hline Type II Plateau & 10 & 52.63 & 2 & 20.0 & 12 & 41.38 & & \\
\hline Type III Wash out & 0 & 0.0 & 8 & 80.0 & 8 & 27.59 & & \\
\hline Total & 19 & $100 \%$ & 10 & $100 \%$ & 29 & $100 \%$ & & \\
\hline
\end{tabular}

Table (3): Lesions' characteristics on diffusion weighted imaging.

\begin{tabular}{|c|c|c|c|c|c|c|}
\hline & \multicolumn{2}{|c|}{ Benign $(\mathrm{n}=19)$} & \multicolumn{2}{|c|}{ Malignant $(\mathrm{n}=10)$} & $\begin{array}{c}\text { Fisher s } \\
\text { exact }\end{array}$ \\
\cline { 2 - 6 } & No & $\mathbf{\%}$ & $\mathbf{N o}$ & $\mathbf{\%}$ & \\
\hline Restricted & 2 & 10.53 & 9 & 90.0 & 15.6 & $<0.001^{*}$ \\
\hline Facilitated & 17 & 89.47 & 1 & 10.0 & \\
\hline
\end{tabular}


Table (4): Apparent diffusion coefficient value of benign \& malignant lesions.

\begin{tabular}{|c|c|c|c|c|c|c|c|c|}
\hline ADC Value & Minimum & Maximum & Mean & Median & sd & T test & P value \\
\hline Benign & 0.86 & 2.4 & 1.4 & 1.4 & 0.45 & 3.58 & $0.001^{*}$ \\
\hline Malignant & 0.56 & 1.4 & 0.85 & 0.74 & 0.29 & \\
\hline
\end{tabular}

N.B The ADC Values are multiplied by $10^{-3} \mathrm{sec} / \mathrm{mm}^{2}$

Table (5): Comparison of Sensitivity, Specificity, \& accuracy of dynamic contrast enhanced magnetic resonance imaging, diffusion weighted imaging with apparent diffusion coefficient $\&$ the combined magnetic resonance imaging protocol.

\begin{tabular}{|c|c|c|c|}
\hline Modality & DCE-MRI & $\begin{array}{c}\text { DWI\& } \\
\text { ADC }\end{array}$ & Combined \\
\hline Sensitivity & 90 & 90 & 90 \\
\hline Specificity & 84.2 & 89.47 & 94.74 \\
\hline PPV & 75 & 81.82 & 90 \\
\hline NPV & 94.12 & 94.44 & 94.74 \\
\hline Accuracy & 86.2 & 89.66 & 93.1 \\
\hline
\end{tabular}

Table (6): Cutoff apparent diffusion coefficient value for diagnosis of malignant lesions.

\begin{tabular}{|c|c|c|c|c|c|c|c|c|c|}
\hline Cutoff Value & AUC & SE & P value & CI & Sensitivity & Specificity & PPV & NPV & Accuracy \\
\hline$<\mathbf{0 . 8 6}$ & $\mathbf{0 . 9 5}$ & $\mathbf{0 . 0 4 8}$ & $<\mathbf{0 . 0 0 1}$ & $\begin{array}{c}\mathbf{4 7 . 3}- \\
\mathbf{9 9 . 7}\end{array}$ & $\mathbf{8 7 . 5}$ & $\mathbf{1 0 0 . 0}$ & $\mathbf{1 0 0 . 0}$ & $\mathbf{9 5 . 0}$ & $\mathbf{9 6 . 5 5}$ \\
\hline
\end{tabular}

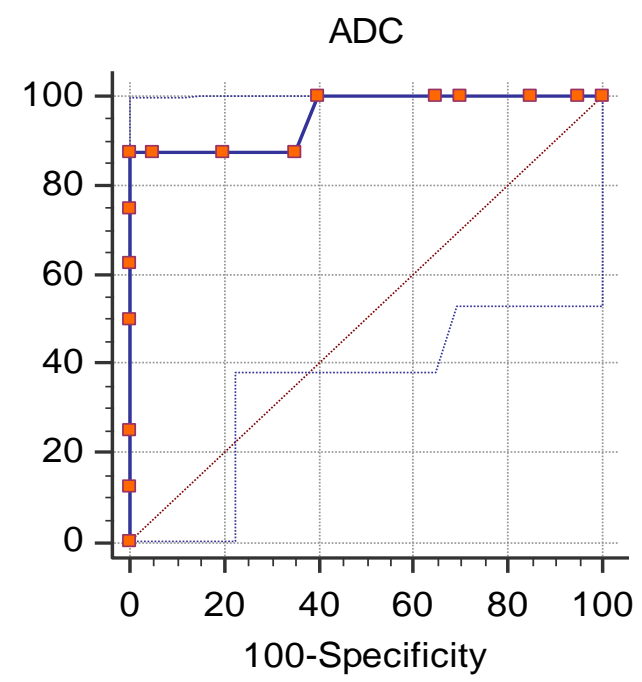

Fig. (1): ROC curve of cutoff ADC value for diagnosis of malignant lesion.

\section{DISCUSSION}

Asymmetrical breast densities can be the only mammographic findings although it may hide an underlying mass. ${ }^{[1]}$

Asymmetry type is seen only in one mammographic view, is usually due to benign causes. ${ }^{[13]}$
Global asymmetry is seen in two mammographic views, it is usually benign too, unless it is associated with other findings, in this case it requires further evaluation. ${ }^{[14]}$

Focal asymmetry must been noticed in two mammographic views. ${ }^{[13]}$

Developing asymmetry is checked in comparison to previous mammography $\&$ it 
must be seen in two mammographic views, developing asymmetry requires special concern as its reported prevalence of malignancy is higher than the other types of asymmetry, which may reach $26.7 \%$ malignancy risk in diagnostic mammography. [14]

DCE- MRI provides better evaluation of the benign appearing findings $\&$ the highly probable malignancy on conventional methods. ${ }^{[1]}$

DWI provides important information about the tissue composition \& its physical characteristics, without the need of contrast agent administration, ADC values vary between malignant \& benign lesions, hence benign lesions usually show facilitated diffusion with higher ADC values, malignant lesions usually demonstrate restricted diffusion with lower ADC value, adding the DWI to the standard MRI breast may increase the overall MRI specificity \& accuracy. ${ }^{[15]}$

This study is performed to evaluate the utility of DCE- MRI \& DWI in assessment of mammographic asymmetric densities in patients classified as BI-RADs (III, IV, \& V). In a duration of 6 months, this study included 29 women with breast complaints, with a mean age of 49.7 years, which (the mean age of the studied group) agrees with Jensen et al., who stated that the mean age of their studied groups for the diagnostic mammography were 49.2 years in United States \& 49.8 years in Denmark. ${ }^{[14]}$

The minimum age of our studied group was 37 years, this agrees with Chan et al., 2013 who stated that the minimum age of their studied symptomatic patients who underwent diagnostic mammography was 35 years. ${ }^{[17]}$

In agreement with Youk et al., \& Berg $\&$ Yang who reported that the prevalence of global asymmetry in mammography is $3 \%$ compared with other types of asymmetry which their prevalences are $\geq 1 \%$ of all mammography. ${ }^{[14,18]}$ In our study we found that, the most common asymmetry type was global asymmetry by $58.6 \%$ followed by $41.4 \%$ with focal asymmetry.

Also in accordance with Youk et al., who stated that Type 1 asymmetry is a benign finding $\&$ it is due to superimposition of normal fibroglandular tissue in $80 \%$ of cases, ${ }^{[18]}$ Asymmetry (one view) wasn't found in our study as this study evaluated asymmetry findings of cases classified on BI-RADS (IIIV).

In disagreement with Moukhtar \& EL Maati who found that 39 out of 110 of the studied lesions $35.45 \%$ were non mass lesions \& 71 out of 110 studied lesions $64.55 \%$ were mass lesions. ${ }^{[19]}$ In our study we found the $41.4 \%$ \& of the studied lesions were non mass lesions \& show non mass like enhancement while the mass lesions were $27.6 \%$, also in additional in our study we found $31 \%$ of lesions show both mass \& non mass like enhancement.

In accordance with Badawi \& Hassan, Mandell, \& Berg \& Yang who stated that, wash out curve presents in $57 \%$ of malignant lesions while fast decrease in the intensity signal by $10 \%$ indicates malignancy in $87 \%$ of cases, ${ }^{[1,20,14]}$ also in agreement with El Bakry et al., who studied 74 lesions, \& reported that $61.1 \%$ of the malignant lesions showed wash out curve \& $25 \%$ of them showed plateau curve. ${ }^{[10]}$ In this study the curve type of $41.38 \%$ of lesions was Plateau (Type II), although (type III) Wash out curve was the least type (27.59\%) among studied group, it has statistical significant with malignancy in this study \& it counts $80 \%$ of the malignant lesions, the rest (20\%) showed Plateau curve (Type II), while plateau curve is shown in $52.6 \%$ \& persistent (Type I) curve in $47.4 \%$ of the benign cases.

The small sample (29 cases) \& the short duration (6 months) of this study made it insufficient to assess the relation between each type of asymmetry \& malignancy risk. However, in this study we found that the most of the asymmetry is caused by benign lesions $65.52 \%$ while $34.48 \%$ are due to malignant causes, the most frequent benign lesion was the fibrocystic changes in $31.58 \%$ of benign cases, \& the most common malignant lesion was invasive ductal carcinoma in $40 \%$ of the malignant cases, These findings agree with Badawi \& Hassan who found that $72 \%$ of asymmetry findings are due to benign lesions $\&$ the most frequent benign lesion was fibrocystic changes in $16.2 \%$, while $27.9 \%$ are caused by malignant lesions, ${ }^{[1]} \&$ also 
agree with leung et al., 2007 who reported that the benign lesions were $60 \%$ of cases \& malignant lesions were $26.7 \%$ of lesions, the most common malignant tumor was IDC, while fibrosis was the most common benign lesion. ${ }^{[3]}$ The slight difference in our study compared to Badawi \& Hassan, \& leung et al.'s findings is thought to be due to the exclusion of BI-RADS II cases in our study .

In disagreement with Mandell who stated that a standard 1.5 tesla MRI in clinical use to evaluate breast cancer has been reported to have a negative predictive value of $98.9 \%$ \& a low positive predictive value for malignancy $49.7 \%,{ }^{[20]}$ In our study the $1.5 \mathrm{~T}$ MRI machine had a positive predictive value of $75 \%$ but the negative predictive value was $94.12 \%$ which was not far from the above authors' percentages.

DCE- MRI in our study has a sensitivity of $90 \%$, a specificity of $84.21 \%$ \& an accuracy of $86.2 \%$ these findings agree with Bluemke et al., who reported that the sensitivity of DCE-MRI ranges from 88$100 \%$ while specificity range is $68-96 \% .{ }^{[6]}$

DWI \& ADC values provide a promising tool in screening \& diagnosis of breast lesions without the use of contrast agents, especially in patients with contraindication to them, thus it may decrease the cost of the examination.

In agreement with El Bakry et al., who reported that $94.4 \%$ of the malignant lesions showed restricted diffusion \& $5.6 \%$ of the malignant lesions showed facilitated diffusion, while $92.1 \%$ of the benign lesions represented facilitated diffusion \& $7.9 \%$ of them revealed restricted diffusion. ${ }^{[10]}$ In our study $89.47 \%$ of the benign lesions showed facilitated diffusion \& $10.53 \%$ of them showed restricted diffusion, while $90 \%$ of malignant lesions showed restricted diffusion, $\& 10 \%$ of them showed facilitated diffusion.

In agreement with El Bakry et al., reported that the PPV for his study was $84.6 \%$ \& the NPV was $91.4 \%,{ }^{[10]}$ in our study we found that the PPV was $81.82 \%$ \& NPV was $94.44 \%$.

Abdulghaffar and Tag-Aldeen, reported that the mean $\mathrm{ADC}$ value for benign lesions in their research was $\left(1.48 \pm 0.33 \times 10^{-}\right.$ $\left.{ }^{3} \mathrm{sec} / \mathrm{mm}^{2}\right)$, \& the ADC range was (1.23- $\left.1.67 \times 10^{-3} \mathrm{sec} / \mathrm{mm}^{2}\right) \&$ the mean ADC value for the malignant lesions was $\left(0.93 \pm 0.27 \times 10^{-}\right.$ $\left.{ }^{3} \mathrm{sec} / \mathrm{mm}^{2}\right)$, \& the ADC range was (0.76$\left.1.29 \times 10^{-3} \mathrm{sec} / \mathrm{mm}^{2}\right),{ }^{[21]}$ also Wahab et al. stated that the mean ADC value for the benign lesions was $\left(1.6 \pm 0.33 \times 10^{-3} \mathrm{sec} / \mathrm{mm}^{2}\right) \&$ the mean ADC value for the malignant lesions was $\left(0.84 \pm 0.25 \times 10^{-3} \mathrm{sec} / \mathrm{mm}^{2}\right) .{ }^{[22]}$

In agreement with the above mentioned studies that show a lower ADC value for malignant lesions than that for benign ones, in our study the mean ADC value for benign lesions was $\left(1.4 \pm 0.45 \times 10^{-}\right.$ ${ }^{3} \mathrm{sec} / \mathrm{mm}^{2}$ ), the median was $1.4 \times 10^{-3} \mathrm{sec} / \mathrm{mm}^{2}$, $\&$ the range was $\left(0.86-2.4 \times 10^{-3} \mathrm{sec} / \mathrm{mm}^{2}\right)$, while the mean ADC value for malignant lesions was $\left(0.85 \pm 0.29 \times 10^{-3} \mathrm{sec} / \mathrm{mm}^{2}\right)$, the median was $\left(0.74 \times 10^{-3} \mathrm{sec} / \mathrm{mm}^{2}\right), \&$ the range was $\left(0.56-1.4 \times 10^{-3} \mathrm{sec} / \mathrm{mm}^{2}\right)$.

In accordance with Periera et al. who reported that the recent studies used different b values ranged $(0-1000) \&$ found a statistical significant difference in ADC values between malignant $\&$ benign tumors with a sensitivity ranged from $81 \%-93 \%$ \& a specificity ranged from $80-88 \%,{ }^{[23]} \&$ in agreement with Abdulghaffar and Tag-Aldeen, who reported higher readings of the sensitivity \& the specificity of their study, which were $95.4 \%$ \& $97.5 \%$ respectively, ${ }^{[21]}$ in our research the sensitivity was $90 \%$, the specificity was $89.4 \%$ \& the accuracy was $89.65 \%$.

In difference with Wahab et al.,who reported that the ADC cutoff value between benign \& malignant lesions was $1.02 \times 10^{-3}$ $\mathrm{sec} / \mathrm{mm}^{2}$ with sensitivity of $90 \%$, the specificity of $95 \%$, PPV of $100 \%$, NPV of $90.4 \% \&$ the accuracy of $92 \%,{ }^{[22]}$ The ROC curve in our study showed that the cutoff ADC value is $<0.86 \times 10^{-3} \mathrm{sec} / \mathrm{mm}^{2}$ with $87.5 \%$ sensitivity, $100 \%$ specificity, $100 \%$ PPV, 95\% NPV \& 96.55\% accuracy, which was lower than the above studies .

In our research by combining the two MRI protocols (DCE-MRI \& DWI) \& comparing their results to histopathological findings, the overall accuracy \& specificity of the MRI increase \& the readings were $90 \%$ sensitivity, 94.74\% specificity, 90\% PPV, 94.74\% NPV \& 93.1\% accuracy, these results agree with El Bakry et al., who reported that by combining both MRI protocols (DCE-MRI 
\& DWI) the validity of MRI has been increased to be $97.2 \%$ sensitivity, $94.7 \%$ specificity, $94.6 \% \mathrm{PPV}, 97.3 \% \mathrm{NPV}, \&$ $95.9 \%$ accuracy. ${ }^{[10]}$

In conclusion, Combining DWI to DCE-MRI in assessment of asymmetric breast densities provided better characterization \& differentiation of underlying lesions especially of probably benign, suspicious, \& highly suggestive malignant findings on conventional imaging, \& the combined MRI protocol increases the overall MRI accuracy.

Recommendations: a better evaluation of each type of asymmetry requires larger sample, longer duration \&/or studying of each type individually.

\section{Declaration of interest}

The authors report no conflicts of interest. The authors alone are responsible for the content and writing of the paper.

Funding information None declared

\section{REFERENCES}

[1] Badawi HA, Hassan AAA (2010): The Role of MRI in assessment of asymmetrical breast densities. The Egyptian Journal of Radiology and Nuclear Medicine; 41, 502.

[2] Samardar P, De Paredes ES, Grimes MM, Wilson JD (2002): Focal Asymmetric Densities Seen at Mammography: US and Pathologic Correlation. Radiological Society of North America Journal ${ }^{\circ}$ RSNA; Volume 22 . Number 1, 20.

[3] Leung JWT, Sickles EA (2007): Developing asymmetry identified on mammography: correlation with imaging outcome and pathologic findings. Am J Roentgenol; 188(3):667-75.

[4] Kuhl CK, Schrading S, Leutner CC, Morakkabati-Spitz N, Wardelmann E, Fimmers R, et al (2005): Mammography, breast ultrasound, and magnetic resonance imaging for surveillance of women at high familial risk for breast cancer. J Clin Oncol; 23: 8469-76.

[5] Pareira FP, Martins G. Carvalhaes de Oliveira RdeV (2011): Diffusion magnetic resonance imaging of the breast. Magn Reson Imaging Clin N Am; 19(1):95-110.

[6] Bluemke DA, Gatsonis CA, Chen MH, DeAngelis GA, DeBruhl N, Harms S, et al (2004): Magnetic resonance imaging of the breast prior to biopsy. JAMA; 292:2735-42.

[7] Bassiouny RH, Youssef T, Hassan O (2012): Diagnostic performance of breast MRI with and without the addition of quantitative diffusion weighted imaging. The Egyptian Journal of Radiology and Nuclear Medicine; 43: 311-323.

[8] Kvistad KA, Rydland J, Vainio J, Smethurst HB, Lundgren S, Fjosne HE, et al (2000): Breast lesions: evaluation with dynamic contrast-enhanced T1- weighted MR imaging and with $\mathrm{T} 2 *$-weighted first pass perfusion MR imaging. Radiology; 216:54553.

[9] Marini C, Iacconi C, Giannelli M, Cilotti A, Moretti M, Bartolozzi C (2007): Quantitative diffusion-weighted MR imaging in the differential diagnosis of breast lesion. EurRadiol; 17(10):2646-55.

[10] EL Bakry MAH, Sultan AA, El-Tokhy NA, Yossif TF, Ali CAA (2015): Role of diffusion weighted imaging and dynamic contrast enhanced magnetic resonance imaging in breast tumors The Egyptian Journal of Radiology and Nuclear Medicine; 46, 791-804.

[11] Hatakenaka M, Soeda H, Yabuuchi H, Matsuo Y, Kamitani T, Oda Y, et al (2008): Apparent diffusion coefficients of breast tumors: clinical application. MagnReson Med Sci; 7(1):23-29.

[12] Al-saadi WI, Shallab EN, Naji S (2015): Diffusion weighted MRI in the characterization of solitary breast mass. The Egyptian Journal of Radiology and Nuclear Medicine; 46, 1337-1341.

[13] Rao AA, Feneis J, Lolande C, OjedaFournier H (2016): A Pictorial Review of Changes in the BI-RADS Fifth Edition. RadioGraphics ;36:623-639. doi.org/10.1148/rg.2016150178

[14] Berg WA, Yang WT (2014): Diagnostic imaging Breast. Second Edition. Salt Lake City, Utah: Amirsys

[15] Ibrahim YA, Habib L, Deif A (2015): Role of quantitative diffusion weighted imaging in characterization of breast masses. The Egyptian Journal of radiology \& nuclear medicine; $46, \quad 805-810$. doi.org/10.1016/j.ejrnm.2015.05.006.

[16] Jensen A, Geller BM, Gard CC, Miglioretti DL, Yankaskas B, Carney BA, et al (2010): Performance of diagnostic mammography differs in the United States and Denmark. Int J Cancer; 127(8): 1905-1912. doi:10.1002/ijc.25198.

[17] Chan VW, Bell MG, and Brown AL (2013): PB.47: Mammography in symptomatic women aged 35 to 39 years. Breast Cancer Research; 15(1):P47.doi.org/10.1186/bcr3547. 
[18] Youk JH, Kim EK, Ko KH, Kim MJ (2009): Asymmetric Mammographic Findings Based on the Fourth Edition of BI-RADS: Types, Evaluation, and Management. RadioGraphics; 29:1, e33-e33. doi.org/10.1148/rg.e33.

[19] Moukhtar FZ, EL Maati AAA (2014): Apparent diffusion coefficient values as an adjunct to dynamic contrast enhanced MRI for discriminating benign \& malignant breast lesion presenting as mass and non-mass like enhancement. The Egyptian Journal of radiology \& nuclear medicine; 45, 597-604.

[20] Mandell J (2013): Core radiology a visual approach to diagnostic imaging. Cambridge university press; 8, 593-657.

[21] Abdulghaffar W, Tag-Aldeen MM (2013): Role of diffusion weighted imaging (DWI) \& apparent diffusion coefficient (ADC) In differentiating between benign \& malignant breast lesions. The Egyptian Journal of radiology \& nuclear medicine; 44, 945-951.

[22] Wahab M A K A, Abdel Kareem H, Hassan EE (2015): The utility of diffusion weighted MRI \& apparent diffusion coefficient in characterization of breast masses. The Egyptian Society of Radiology and Nuclear Medicine;46:1257-1265.

doi.org/10.1016/j.ejrnm.2015.06.016.

[23] Pereira FP, Martins G, Figueiredo E, Domingues $\mathrm{MN}$, Domingues $\mathrm{RC}$, Da Fonesca LM, et al (2009): Assessment of breast lesions with diffusion -weighted MRI: comparing the use of different $b$ values. American Journal of Roentgenol;193(4):10301035.

To Cite This Article: Mousa HM, Abdulmonaem GE, and El Sammak DE, Role of Diffusion Weighted Imaging \& Dynamic Contrast Enhanced Magnetic Resonance Imaging in Assessment of Asymmetric Breast Densities, ZUMJ 2020; 26 (2):335-343, Doi: 10.21608/zumj.2019.11327.1183 